\title{
Target Detection in Three-Dimension Sensor Networks Based on Clifford Algebra
}

\author{
Tiancheng HE, Weixin XIE, Wenming CAO \\ Institute of Intelligent Information System, Shenzhen University, Shenzhen, China \\ Email: sd76130201@szu.edu.cn \\ Received April 27, 2009; revised May 8, 2009; accepted May 9, 2009
}

\begin{abstract}
The three-dimensional sensor networks are supposed to be deployed for many applications. So it is significant to do research on the problems of coverage and target detection in three-dimensional sensor networks. In this paper, we introduced Clifford algebra in 3D Euclidean space, developed the coverage model of 3D sensor networks based on Clifford algebra, and proposed a method for detecting target moving. With Clifford Spinor, calculating the target moving formulation is easier than traditional methods in sensor node's coverage area.
\end{abstract}

Keywords: 3D Sensor Networks, Clifford Algebra, Spinor, Target Detection, Coverage

\section{Introduction}

Three-dimensional sensor networks [1] could enable a broad range of applications: Video Surveillance, Ocean Sampling, Environmental Monitoring, Assisted Navigation, and etc. As an emerging technology which can put the information field to a new stage, the theory and applications of three-dimensional intelligent sensor networks have become a key research aspect. With the coming availability of low cost, short range radios along with advances in wireless networking, it is expected that wireless ad hoc sensor networks will become commonly deployed. So it is useful to study three-dimensional intelligent sensor network systems. The coverage problem and target detection problem are the fundamental issues in 3D intelligent sensor network systems. Studies on sensor networks include distributed network, distributed information acquisition, distributed intelligent information fusion and so on. Xie [2,3] proposed a coverage analysis approach for sensor networks based on Clifford algebra. In a 2-dimensional plane, a homogeneous computational method of distance measures has been provided for points, lines and areas, and a homogeneous coverage analysis model also has been proposed for targets with hybrid types and different dimensions. Thus, an analysis framework has been established for sensor net- works in Clifford geometric space. To evaluate the quality of network coverage, Megerian [4] used Voronoi diagram and Delaunay triangulation respectively to define the worst and best-case coverage in sensor networks. There are also a lot of improved methods to solve these problems [5-8].

The target detection problem in sensor networks also has been a topic of extensive study under different metrics and assumptions [9-11]. There are already some related theories and algorithms proposed for solving the problems of target detection in sensor networks. The work of target detection in sensor networks includes many aspects, as the following:

The Traversing Path Detection [12]: A traversing path without being detected should not intersect the sensing areas of any sensors [13]. The detection rate of a sensor network is interested in application scenarios such as vehicles crossing a battlefield in military operations. Meguerdichian mentioned a novel coverage model for the target detection [14], and proposed an approximate value algorithm for calculating the traversing path [15]. There are also some distributed algorithms to calculate the efficient value in the sensor networks for detecting target [16]. Another way to solve the problem is the localized algorithm with lower computational complexity [17]. It uses polar coordinates to detect target and calcu- 
late the path, and refers the Euler equation to calculate the minimal exposure traversing path.

The Exposure of Target [18]: The exposure of target detection is another aspect of the related work. The most popular definition is the information exposure to the estimation of target parameters [18]. With the information exposure formulation and grid graph, the minimal exposure traversing path in detecting target could be achieved. Meanwhile, some heuristic algorithms were also proposed for nodes deployment according to the degree of information exposure [8,15].

The Deployment of Nodes Density [19]: The nodes density is used for ensuring the probability of target detection[19]. It is assumed that the motive target could traverse the deployed area of sensor networks with a fixed velocity and a line path. The studies for deployment of nodes density include the probability sensor model and exposure model [20], grid deployment and random deployment in wireless sensor networks [21], and The critical nodes density based on continuum percolation [22]. The all-sensor field intensity can be modeled as a two dimensional Poisson shot noise process for large-scale sensor networks under the general sensing model [23].

Barrier Coverage [24]: Barrier coverage was proposed by Kumar [24] who mentioned it as an appropriate notion of coverage when a sensor network is deployed to detect targets traversing a protected region, which represents a promising and popular class of applications for wireless sensor networks. There are also some studies for the problem such as minimum segment barrier coverage [25], and double barrier coverage [11].

The coverage and target detection problem can be solved optimally in 2D plane by dividing the polygon into non-overlapping triangles, but it becomes NP-hard in 3D space. In this paper, we present a method for calculating target moving in 3D sensor networks with the coverage analysis approach based on Clifford algebra, which establishes a coordinate-free, homogeneous coverage model for different dimensional spaces and targets with hybrid types. This approach gives the intact relative geometric description between sensor node and target. With the Clifford Spinor, calculating target moving formulation will be more simply and effectively than traditional method for sensor networks.

The paper is organized as follows. In Section 2, we state relevant background of Clifford algebra. In Section 3 , we present our model and target moving formulation. In Section 4, the algorithm based on Clifford algebra is proposed. In Section 5, we present our conclusions.

\section{Clifford Algebra in 3D Euclidean Space}

Rectangular Cartesian coordinate system is adopted in 2D Euclidean space, and any vector a can be written as $\mathbf{a}=a_{1} e_{1}+a_{2} e_{2}$, where $e_{1}, e_{2}$ are the unit vectors of $x, y$ directions, respectively. Hereby the geometric product between vector $\mathbf{a}$ and $\mathbf{b}$ can be calculated as:

$$
\begin{aligned}
\mathbf{a b} & =\left(a_{1} e_{1}+a_{2} e_{2}\right)\left(b_{1} e_{1}+b_{2} e_{2}\right) \\
& =a_{1} b_{1}+a_{2} b_{2}+a_{1} b_{2} e_{1} e_{2}+a_{2} b_{1} e_{2} e_{1}
\end{aligned}
$$

Here $e_{1} e_{2}=e_{2} e_{1}=1$. If we appoint $e_{1} e_{2}$ as the area unit with direction, and let $e_{1} e_{2}=-e_{2} e_{1}$, so the geometric product is

$$
\begin{aligned}
\mathbf{a b} & =a_{1} b_{1}+a_{2} b_{2}+\left(a_{1} b_{2}-a_{2} b_{1}\right) e_{1} e_{2} \\
& =\mathbf{a} \bullet \mathbf{b}+\mathbf{a} \wedge \mathbf{b}
\end{aligned}
$$

$\mathbf{a} \bullet \mathbf{b}$ is inner product, and $\mathbf{a} \wedge \mathbf{b}$ is outer product. The inner product is always coincident with dot product in vector algebra, while outer product is the measurement of parallelogram area composed by adjacent borders $\mathbf{a}$, b. If a is rotated counter-clockwise with an angle which is no more than $\pi$ to overlap $\mathbf{b}$, the measurement of parallelogram area is larger than zero. Otherwise it is less than zero. The area uses $e_{1} e_{2}$ as a measurement unit, and its absolute value is $\mathbf{a b} \sin \theta$, where $\theta$ is the angle between $\mathbf{a}$ and $\mathbf{b}$. Here $\mathbf{a} \bullet \mathbf{b}=\mathbf{a b} \cos \theta$.

Suppose that $e_{1} e_{2}=i$, then $e_{2} e_{1}=-i$, and

$$
i^{2}=e_{1} e_{2} e_{1} e_{2}=-e_{1} e_{1} e_{2} e_{2}=-1
$$

Because $e_{1} e_{2}=-e_{2} e_{1}$, exchanging the position of $e_{1} e_{2}$ will appear a minus, and exchanging the position $n$ times should multiply (-1)n. We call it negative exchange, and should take care of it in Clifford algebra.

After confirming the $e_{1} e_{2}=i$, we will have

$$
\begin{gathered}
e_{1} i=e_{1} e_{1} e_{2}=e_{2}, \quad e_{2} i=e_{2} e_{1} e_{2}=-e_{1} e_{2} e_{2}=-e_{1}, \\
\mathbf{a} i=\left(a_{1} e_{1}+a_{2} e_{2}\right) e_{1} e_{2}=a_{1} e_{2}-e_{2} e_{1}
\end{gathered}
$$

So each vector multiplies $i$ on right side to let the vector be rotated counter-clockwise with $\pi / 2$. And

$$
\begin{aligned}
\mathbf{a} e^{i \theta} & =\left(a_{1} e_{1}+a_{2} e_{2}\right)(\cos \theta+i \sin \theta) \\
& =a_{1} e_{1} \cos \theta+a_{1} e_{1} i \sin \theta+a_{2} e_{2} \cos \theta+a_{2} e_{2} i \sin \theta \\
& =\left(a_{1} \cos \theta-a_{2} i \sin \theta\right) e_{1}+\left(a_{1} \sin \theta+a_{2} \cos \theta\right) e_{2}
\end{aligned}
$$

It definitely describes that the ordinate unit vectors $e_{1} e_{2}$ are fixed, vector $\mathbf{a}$ is rotated counter-clockwise with $\theta$. It is obvious that $i$ is not only just as -1 , but also has specific geometric significant. The aze $e^{i \theta}$ also denotes that $\mathbf{a}$ is rotated with $\theta$, and magnified $z$ times. $z$ is the module of complex number.

The nodes in sensor networks need scalar and directed quantity to be described together, so we use Equation (1) for calculating. The quantity $\mathbf{a} \wedge \mathbf{b}$ is called dual vector 
or bivector, its unit is $e_{1} e_{2}=i=e_{1} \wedge e_{2}$. According to (1), $e_{1} e_{2}=e_{1} \bullet e_{2}+e_{1} \wedge e_{2}=e_{1} \wedge e_{2}$ due to $e_{1} \bullet e_{2}=0$.

The vector a in $3 \mathrm{D}$ Euclidean space $\mathfrak{R}^{3}$ can be written as $\mathbf{a}=a_{1} e_{1}+a_{2} e_{2}+a_{3} e_{3}$, where $e_{1}, e_{2}, e_{3}$ are orthonormal vectors. So

Definition 1: The geometric product of vector a and b in $3 \mathrm{D}$ space is

$$
\begin{aligned}
\mathbf{a b}= & \left(a_{1} e_{1}+a_{2} e_{2}+a_{3} e_{3}\right)\left(b_{1} e_{1}+b_{2} e_{2}+b_{3} e_{3}\right) \\
= & a_{1} b_{1}+a_{2} b_{2}+a_{3} b_{3}+\left(a_{1} b_{2}-a_{2} b_{1}\right) e_{1} e_{2} \\
& +\left(a_{2} b_{3}-a_{3} b_{2}\right) e_{2} e_{3}+\left(a_{3} b_{1}-a_{1} b_{3}\right) e_{3} e_{1} \\
= & \mathbf{a} \bullet \mathbf{b}+\mathbf{a} \wedge \mathbf{b}
\end{aligned}
$$

Let $e_{1} e_{2} e_{3}=i$, and $e_{1} e_{2}=e_{1} e_{2} e_{3} e_{3}=i e_{3}, e_{2} e_{3}=i e_{1}$, $e_{3} e_{2}=i e_{2}$. So

$$
\begin{aligned}
\mathbf{a} \wedge \mathbf{b}= & \left(a_{1} b_{2}-a_{2} b_{1}\right) i e_{3}+\left(a_{2} b_{3}-a_{3} b_{2}\right) i e_{1} \\
& +\left(a_{3} b_{1}-a_{1} b_{3}\right) i e_{2} \\
= & i(\mathbf{a} \times \mathbf{b})
\end{aligned}
$$

where $\mathbf{a} \times \mathbf{b}$ is the scalar product in vector algebra.

Suppose that $i e_{k}=i_{k}(k=1,2,3), i_{1}=e_{2} e_{3}, i_{2}=e_{3} e_{1}$, $i_{3}=e_{1} e_{2}$, and the $3 \mathrm{D}$ vector $i_{k}$ is the product of the pseudo-scalar $i$ and the three basis vectors $e_{k}$. Here $i_{1} e_{2}=e_{2} e_{3} e_{2}=-e_{3}$, and $e_{2} i_{1}=e_{2} e_{2} e_{3}=e_{3}=-i_{1} e_{2}$. The $i$ is direct volume unit in $3 \mathrm{D}$ space.

In $3 \mathrm{D}$ space, any rotation is denoted as the result of two vector reflections because the vectors are not always coplanar. The vector $x_{1}$ is from the reflection of vector $x$ with the plane I whose normal is basis vector $u$, depicted in figure 1 . And the vector $x^{\prime}$ is from the reflection of vector $x_{1}$ with the plane II whose normal is basis vector $v$. So vector $x$ rotates to $x^{\prime}$ with the angle $\theta$, and $\theta=2 \alpha$ where $\alpha$ is the angle between $u$ and $v$. With Clifford algebra, it is shown as

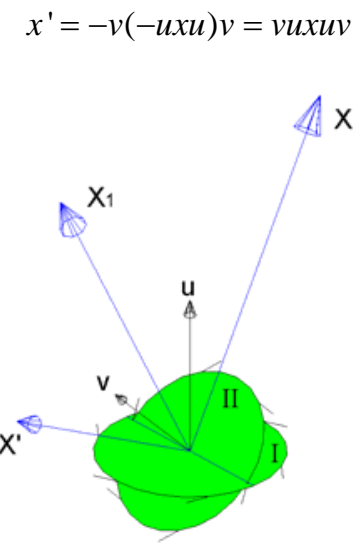

Figure 1. Vector rotation in 3D space.
Let $R=u v$, so

$$
x^{\prime}=R^{T} \times R
$$

where $R^{T}$ is the reversion of $R, R$ is called Spinor [26] which is composed of scalar $u \cdot v$ and bivector $u \wedge v$. In general

$$
\begin{aligned}
R & =u \cdot v+u \wedge v=u \cdot v+i(u \times v) \\
& =\cos \alpha+i n \sin \alpha \\
& =e^{i n \alpha}
\end{aligned}
$$

where $n$ is the basis vector whose direction is decided by $u \times v . \alpha$ is the angle between $u$ and $v$. $R=e^{i n \alpha}$ can be written as $R=\alpha+i b$, where $\alpha$ is scalar and $b$ is vector, here $\alpha^{2}+b^{2}=1$. Any bivector can be written as the product of $i$ and a vector due to $e_{1} e_{2}=i e_{3}$, so $i b$ is a bivector. $R^{T}=e^{-i n \alpha} \quad$ if $R=e^{i n \alpha}$ while $R^{T}=\alpha-i b$ if $R=\alpha+i b$ and $R^{T}=v u$ if $R=u v$. That means $R R^{T}=R^{T} R=1 . R=e^{i n \theta / 2}$ if the angle between $x$ and $x^{\prime}$ is $\theta$.

\section{Target Detection in 3D Sensor Networks}

Coverage analysis in sensor networks is essentially to determine whether an arbitrary point in a space is perceived by sensor nodes. Previous methods use models of different dimensional geometric targets to determine whether the interested points covered or not in different dimensional space, respectively. For sensor networks with hybrid types of targets, those methods cannot provide a homogeneous and effective coverage analysis in different dimensional subspaces. The geometric operation in Clifford algebra is independent of coordinates with a determinate dimension. Hence, Xie [2] proposed a coverage model based on the rotation operator in 3D space for sensor networks.

Definition 2: Set an omnidirectional sensor node $S=\left(\mathbf{v}_{s}, \rho e_{1} \wedge e_{2} \wedge e_{3}\right)$ with perceived radius $\rho$, the coverage range of $S$ is $\mathbf{D}=\{x \mid\|x-S\| \leq \rho\}$, here vector $\mathbf{v}_{s} \in S$, so the coverage discrimination model for task region $\mathbf{B}$ in space $G_{3}$ is given by

$$
C(\mathbf{B})= \begin{cases}1, & \forall x \in \mathbf{B},\|\mathbf{R}\|_{x} \leq 1 \\ 0, & \forall x \in \mathbf{B},\|\mathbf{R}\|_{x}>1\end{cases}
$$

where the Spinor $\mathbf{R}=x / \mathbf{v}_{s}$. Figure 2 illustrates the coverage model. 


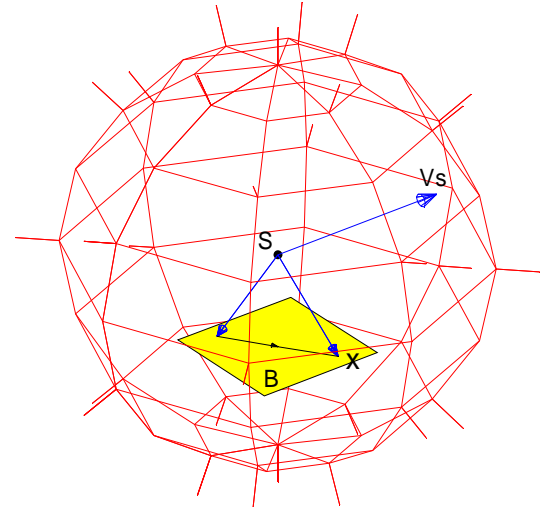

Figure 2. Coverage model based on Clifford Spinor in 3D space.

The relationship between sensor node and target is always depicted by Euler angle, and it would be illustrated by Clifford algebra without coordinates. So the Clifford algebra can present $3 \times 3$ Spinor matrix, and the angle transformation is

$$
\begin{aligned}
\mathbf{R} x & =R_{\phi}^{T} R_{\theta}^{T} R_{\varphi}^{T} x R_{\varphi} R_{\theta} R_{\phi} \\
& =e^{-i e_{3} \phi / 2} e^{-i e_{3} \theta / 2} e^{-i e_{3} \varphi / 2} x e^{i e_{3} \varphi / 2} e^{i e_{3} \theta / 2} e^{i e_{3} \phi / 2}
\end{aligned}
$$

Let $f_{k}=\mathbf{R} \mathrm{e}_{k}$, and $f_{k}$ is the new vector that is isomorphic with $\mathrm{e}_{k}$ in new position after transformation.

Theorem 1: The vector $\xi$ which is perpendicular with axis rotates to $\xi^{\prime}$, so

$$
\xi^{\prime}=\mathbf{R} \xi=R^{T} \xi R=\xi R^{2}
$$

Proof: $\xi$ is perpendicular with vector $b$ in $R=$ $\alpha+i b$ whose direction is same as axis, so $b \xi=b \cdot \xi+$ $b \wedge \xi=b \wedge \xi=-\xi \wedge b=-\xi b$, that means the negative exchange between vectors which are perpendicular with each other. So

$$
R_{j k}=\left[\begin{array}{c}
\cos \varphi \cos \phi-\sin \varphi \cos \theta \sin \phi \\
-\cos \varphi \sin \theta \sin \phi-\sin \varphi \cos \phi \\
\sin \theta \sin \phi
\end{array}\right.
$$

With the method of Clifford algebra, we can easily calculate the $3 \times 3$ Spinor matrix of arbitrary angle $\theta$ rotating around any vector $n$. The element of this matrix is

$$
R_{j k}=f_{j} \bullet e_{k}=\left(R^{\prime} e_{j} R\right) \bullet e_{k}
$$

Generally, it is more suitable that the element under this

$$
\begin{aligned}
\xi^{\prime} & =R^{T} \xi R=(\alpha-i b) \xi(\alpha+i b) \\
& =(\alpha-i b)(\xi \alpha+i \xi b) \\
& =\alpha^{2} \xi-i \alpha b \xi+i \alpha \xi b+b \xi b \\
& =\xi\left(\alpha^{2}+i \alpha b+i \alpha b-b^{2}\right) \\
& =\xi(\alpha+i b)(\alpha+i b)=\xi R^{2}
\end{aligned}
$$

Here the exchange among vector $\xi$, pseudo-scalar $i$ and $\alpha$ is positive, but is negative between $\xi$ and vector $b$.

With $\xi^{\prime}=\xi R^{2}$, consider $R_{\phi}^{T} R_{\theta}^{T} R_{\varphi}^{T} e_{1} R_{\varphi} R_{\theta} R_{\phi}$, here

$$
\begin{aligned}
R_{\varphi}^{T} e_{1} R_{\varphi} & =e_{1} R_{\varphi}{ }^{2}=e_{1} e^{i e_{3} \varphi} \\
& =e_{1}\left(\cos \varphi+i e_{3} \sin \varphi\right) \\
& =e_{1} \cos \varphi+e_{2} \sin \varphi
\end{aligned}
$$

And

$$
\begin{aligned}
& R_{\theta}^{T}\left(e_{1} \cos \varphi+e_{2} \sin \varphi\right) R_{\theta} \\
& \quad=e_{1} \cos \varphi+e_{2} \sin \varphi e^{i e_{1} \theta} \\
& \quad=e_{1} \cos \varphi+e_{2} \sin \varphi \cos \theta+e_{3} \sin \varphi \sin \theta
\end{aligned}
$$

So

$$
\begin{aligned}
f_{1}= & R_{\phi}^{T}\left(e_{1} \cos \varphi+e_{2} \sin \varphi \cos \theta+e_{3} \sin \varphi \sin \theta\right) R_{\phi} \\
= & e_{1} \cos \varphi\left(e^{i e_{3} \phi}\right)+e_{2} \sin \varphi \cos \theta\left(e^{i e_{3} \phi}\right)+e_{3} \sin \varphi \sin \theta \\
= & e_{1} \cos \varphi \cos \phi+e_{2} \cos \varphi \sin \phi+e_{2} \sin \varphi \cos \theta \cos \phi \\
& -e_{1} \sin \varphi \cos \theta \sin \phi+e_{3} \sin \varphi \sin \theta
\end{aligned}
$$

Meanwhile

$$
\begin{aligned}
f_{2}= & e_{2} \cos \varphi \cos \theta \cos \phi-e_{1} \cos \varphi \sin \theta \sin \phi-e_{1} \sin \varphi \cos \phi \\
& -e_{2} \sin \varphi \sin \phi+e_{3} \cos \varphi \sin \theta
\end{aligned}
$$

$$
f_{3}=e_{3} \cos \theta-e_{2} \sin \theta \cos \phi+e_{1} \sin \theta \sin \phi
$$

The Spinor matrix $R_{j k}=\left(f_{j} \cdot e_{k}\right)$ is

$$
\left.\begin{array}{cc}
\cos \varphi \sin \phi+\sin \varphi \cos \theta \cos \phi & \sin \varphi \sin \theta \\
\cos \varphi \cos \theta \cos \phi-\sin \varphi \sin \phi & \cos \varphi \sin \theta \\
-\sin \theta \cos \phi & \cos \theta
\end{array}\right]
$$

circumstance can be written as $R_{j k}=<\left(R^{T} e_{j} R\right) \bullet e_{k}>_{0}$. Here $<>_{0}$ denotes the vector of grade zero which is scalar part, because the result will normally be $<>_{0}+<>_{1}+<>_{2}+<>_{3}$. We have known that the established $R_{j k}$ just is a simple scalar quantity, so we don't have to denote its vector of grade zero, where 


$$
\begin{aligned}
R^{T} e_{j} R & =(\alpha-i b) e_{j}(\alpha+i b) \\
& =(\alpha-i b)\left(\alpha e_{j}+i e_{j} b\right) \\
& =\alpha^{2} e_{j}-i \alpha b e_{j}+i \alpha e_{j} b+b e_{j} b
\end{aligned}
$$

With

$$
\begin{aligned}
& i \alpha\left(e_{j} b-b e_{j}\right)=2 i \alpha\left(e_{j} \wedge b\right)=2 i \alpha i\left(e_{j} \times b\right)=-2 \alpha\left(e_{j} \times b\right), \\
& b e_{j} b=b \bullet\left(e_{j} \bullet b+e_{j} \wedge b\right)+b \wedge\left(e_{j} \bullet b+e_{j} \wedge b\right) \\
&=b \bullet e_{j} b-b \bullet b e_{j}+e_{j} \bullet b b=2 b \bullet e_{j} b+b^{2} e_{j}
\end{aligned}
$$

Hence

$$
R^{T} e_{j} R=\left(\alpha^{2}-b^{2}\right) e_{j}-2 \alpha\left(e_{j} \times b\right)+2 b \bullet e_{j} b,
$$

And
$\left(R^{T} e_{j} R\right) \bullet e_{j}=\left(\alpha^{2}-b^{2}\right) \delta_{j k}-2 \alpha\left(e_{j} \times b\right) \bullet e_{j}+2\left(b \bullet e_{j}\right)\left(b \bullet e_{k}\right)$ where $\alpha=\cos \frac{\theta}{2}, b=n \sin \frac{\theta}{2}, \theta$ is the angle, $n$ is the unit vector for rotating. (3.10) can be written as

$$
R_{j k}=\cos \theta \bullet \delta_{j k}-\sin \theta\left(e_{j} \times n\right) \bullet e_{k}+(1-\cos \theta) n_{j} n_{k}
$$

Here $\delta_{j k}$ is Kronecker symbol. When $j=k, \delta_{j k}=1$, otherwise $\delta_{j k}=0$. The second part in (15) is a general vector operation which can be easily calculated by specific $j, k$ because $\left(e_{j} \times n\right) \bullet e_{k}=-\left(e_{j} \times e_{k}\right) \bullet n$. For example, $j=2, k=3$, and $e_{2} \times e_{3}=e_{1}$, so $\left(e_{j} \times e_{k}\right) \bullet n=$ $e_{1} \bullet n=n_{1}$. Therefore, the second part is $n_{1} \sin \theta$ here which can infer the other parts. The final result is

$$
R_{j k}=\left[\begin{array}{ccc}
\cos \theta+n_{1}^{2}(1-\cos \theta) & n_{1} n_{2}(1-\cos \theta)+n_{3} \sin \theta & n_{1} n_{3}(1-\cos \theta)-n_{2} \sin \theta \\
n_{1} n_{2}(1-\cos \theta)-n_{3} \sin \theta & \cos \theta+n_{2}^{2}(1-\cos \theta) & n_{2} n_{3}(1-\cos \theta)+n_{1} \sin \theta \\
n_{3} n_{1}(1-\cos \theta)+n_{2} \sin \theta & n_{3} n_{1}(1-\cos \theta)-n_{1} \sin \theta & \cos \theta+n_{3}^{2}(1-\cos \theta)
\end{array}\right]
$$

The conventional methods can also achieve this result, but this method is straight and do not need the graph for help. Hence the target moving can be detected in coverage area of sensor networks by Clifford algebra, as shown in figure 3. If the target moves from $x$ to $x^{\prime}$, that is

$$
x^{\prime}=R^{T} x R+a=\mathbf{R} x+a
$$

where $x$ is the position of target.

Each element in (16) would be time function, so the target moving formulation is

$$
\dot{x}^{\prime}=\dot{\mathbf{R}} x+\mathbf{R} \dot{x}+\dot{a}
$$

Theorem 2: In (17), $\dot{\mathbf{R}} x=\dot{R}^{T} x R+R^{T} x \dot{R}$ can be calculated by (18):

$$
\dot{\mathbf{R}} x=R^{T}(\omega \times x) R
$$

where $\omega$ is target's angular velocity to sensor node.

Proof: Suppose that

$$
\dot{R} x=\frac{1}{2} R \Omega
$$

And

$$
\begin{gathered}
\dot{R}^{T} R+R^{T} \dot{R}=0 \\
\frac{1}{2} \Omega^{T} R^{T} R+\frac{1}{2} R^{T} \dot{R} \Omega=0 \\
\Omega^{T}=-\Omega, \dot{R}^{T}=-\frac{1}{2} \Omega R^{T}
\end{gathered}
$$

Here $R^{T} R=1, \dot{R}^{T}=(\dot{R})^{T} . \Omega$ is bivector, and can be denoted as $\Omega=i \omega$. So $\Omega=2 R^{T} \dot{R}$ or $i \omega=2 R^{T} \dot{R}$.

Thus

$$
\dot{\mathbf{R}} x=\dot{R}^{T} x R+R^{T} x \dot{R}=-\frac{1}{2} \Omega R^{T} x R+\frac{1}{2} R^{T} x R \Omega=\frac{1}{2} R^{T}\left(-R \Omega R^{T} x+x R \Omega R^{T}\right) R
$$

and

$$
\dot{\mathbf{R}} x=R^{T} \frac{1}{2}(x \Omega-\Omega x) R=\mathbf{R} x \cdot \Omega=R^{T}(x \cdot \Omega) R
$$

Or

$$
\dot{\mathbf{R}} x=R^{T} \frac{i}{2}(x \omega-\omega x) R=R^{T}(\omega \times x) R
$$

With $\omega \wedge x=i(\omega \times x)$ and $\omega \times x=-i(\omega \wedge x)$,

$$
\dot{x}^{\prime}=R^{T}[(\omega \times x)+\dot{x}] R+\dot{a}
$$

The target moving formulation can further be denoted as

$$
\ddot{x}^{\prime}=\dot{\mathbf{R}}[(\omega \times x)+\dot{x}]+\mathbf{R}[(\dot{\omega} \times x)+(\omega \times \dot{x})+\ddot{x}]+\ddot{a}
$$




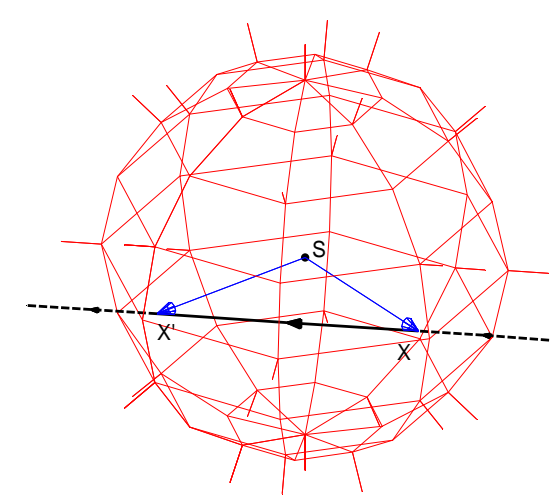

Figure 3. The target moving path detected by sensor node.

where

$$
\begin{aligned}
\dot{\mathbf{R}}(\omega \times x) & =\dot{R}^{T}(\omega \times x) R+R^{T}(\omega \times x) \dot{R} \\
& =-\frac{1}{2} i \omega^{\prime} R^{T}(\omega \times x) R+\frac{1}{2} R^{T}(\omega \times x) R i \omega^{\prime} \\
& =R^{T} \frac{i}{2}\left[(\omega \times x) R \omega^{\prime} R^{T}-R \omega^{\prime} R^{T}(\omega \times x)\right] R \\
& =R^{T} \frac{i}{2}[(\omega \times x) \omega-\omega(\omega \times x)] R \\
& =R^{T} \omega \times(\omega \times x) R \\
& =R^{T}(x \cdot \Omega) \cdot \Omega R
\end{aligned}
$$

And

$$
\dot{\mathbf{R}} \dot{x}=\dot{R}^{T} \dot{x} R+R^{T} \dot{x} \dot{R}=R^{T}(\omega \times \dot{x}) R=R^{T} \dot{x} \cdot \Omega R .
$$

Therefore,

$$
\ddot{x}^{\prime}=R^{T}[\ddot{x}+2(\omega \times \dot{x})+\omega \times(\omega \times x)+\dot{\omega} \times x] R+\ddot{a}
$$

In the 3D space, the movement formulation of the target $m$ is

$$
m \ddot{x}{ }^{\prime}=g^{\prime},
$$

where is the power of the target so

$$
\begin{gathered}
R^{T} m[\ddot{x}+2(\omega \times \dot{x})+\omega \times(\omega \times x)+\dot{\omega} \times x] R+m \ddot{a}=g^{\prime} \\
m[\ddot{x}+2(\omega \times \dot{x})+\omega \times(\omega \times x)+\dot{\omega} \times x]+R m \ddot{a} R^{T}=R g^{\prime} R^{T}=g \\
m \ddot{x}=g-m[2(\omega \times \dot{x})+\omega \times(\omega \times x)+\dot{\omega} \times x]-R m \ddot{a} R^{T}
\end{gathered}
$$

We can achieve the movement formulation of the target in sensor networks, which will help us to analyze the state of the detected target.

\section{Algorithm}

With the movement formulation of the target in 3D sensor networks, we propose a motive target detection algorithm based on Clifford Spinor. We assume that the mo- tive target in sensor networks can be detected by some nodes, and its positions in the range of these nodes also can be calculated. Because the surveillance range of a node is always small, the track of the target in the range will be considered as a line approximately. Our algorithm for tracking the motive target is following:

1) Detect the two vertexes of the track when the target is in the range of node;

2) Use (17) or (20) to calculate the movement formulation of the target;

3) Use the movement states of target to estimate the direction and velocity so as to inform the next correlative node turning on and starting to detect the target;

4) Collect all information from each node, and achieve the track of the motive target in the sensor networks.

Figure 4 shows the motive target detection using moving formulation calculated by Clifford Spinor in 3D sensor networks, where (a) is the target's actual path to traverse sensor networks, and (b) is the target's traversing path achieved by moving formulation. The target positions in sensor's coverage area can be used to calculate the moving formulation to get the traversing path in this area. Connecting all of these paths which are achieved from each node can get the target's track in sensor networks. It is helpful for target tracking and forecasting.

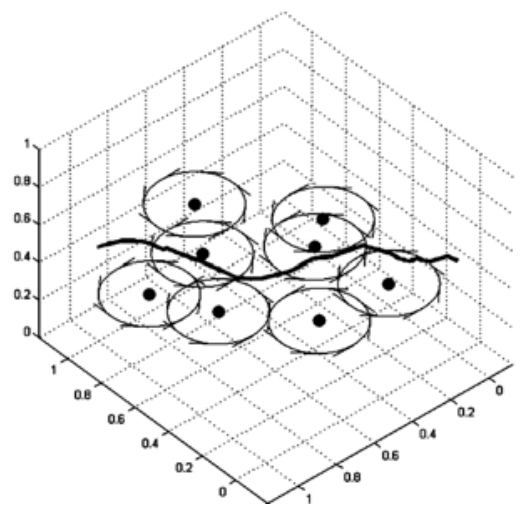

(a)

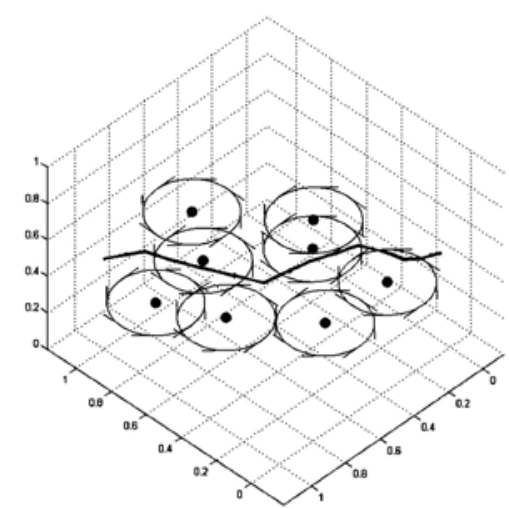

(b)

Figure 4. Target detection in 3D sensor networks. 


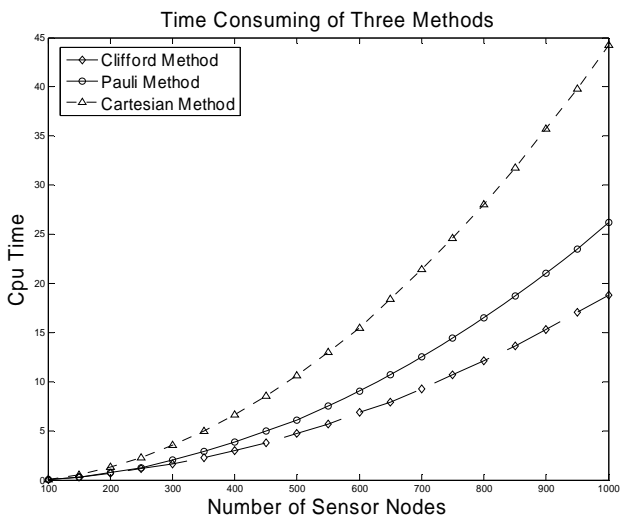

Figure 5. The comparison of time consuming among three methods.

Figure 5 denotes the time consuming to calculate target moving formulation using Cartesian method, polar coordinates method and Clifford method, respectively. It is obvious that the time consuming using Clifford method is lower than that of the other two methods when the number of sensor nodes increases. This is because the Cartesian method should use the information of three axes in 3D space and the polar coordinates method would calculate three $3 \times 3$ Pauli matrices. The quantity of calculation is decreased in Clifford method due to just using Spinor equation to get the moving formulation without axes information in 3D space.

\section{Conclusions}

In this paper, we developed the coverage model of 3D sensor networks with the coverage analysis approach based on Clifford algebra, which establishes a coordinate-free, homogeneous coverage model for different dimensional spaces and targets with hybrid types, and proposed the method for detecting target moving. With Clifford Spinor, calculating the target moving formulation is easier than traditional methods in sensor node's coverage area. It is helpful to the research of coverage analysis in sensor networks.

\section{Acknowledgements}

This research is supported by National Natural Science Foundation (No.60872126) and Guangdong Province Natural Science Foundation (No.8151806001000002).

\section{References}

[1] C. Tian, W. Liu, J. Jin, Y. Wang, and Y. Mo, "Localization and synchronization for 3D underwater acoustic sensor network,” Proceedings of UIC 2007, pp. 622-631,
July 2007.

[2] W. X. Xie, W. M. Cao, and S. Meng, "Coverage analysis for sensor networks based on Clifford algebra," Science in China Series F-Information Sciences, Vol. 51, No. 5, pp. 460-475, 2008.

[3] W. X. Xie, T. C. He, and W. M. Cao, "Path analyses of sensor networks based on Clifford algebra,” Acta Electronica Sinica, Vol. 35, No. 12A, pp. 27-31, 2007.

[4] S. Megerian, F. Koushanfar, M. Potkonjak, M. B. Srivastava, "Worst and best-case coverage in sensor networks,” IEEE Transactions on Mobile Computing, Vol. 4, No. 1, pp. 84-92, 2005.

[5] X. Li, P. Wan, and O. Frieder, "Coverage problem in wireless ad-hoc sensor networks," IEEE Transactions on Computers, Vol. 52, No. 6, pp. 753-763, 2003.

[6] T. C. He, W. M. Cao, and W. X. Xie, "Research of breach issue for sensor networks based on Clifford geometrical algebra," Chinese Journal of Scientific Instrument, Vol. 28, No. 8, pp. 161-165, 2007.

[7] A. D. Gupta, A. Bishnu, and I. Sengupta, "Optimisation problems based on the maximal breach path measure for wireless sensor network coverage,” ICDCIT, pp. 27-40, 2006.

[8] G. Veltri, Q. Huang, G. Qu, and M. Potkonjak, “Minimal and maximal exposure path algorithms for wireless embedded sensor networks,” Proceedings of the 1st International Conference on Embedded Networked Sensor Systems (SenSys'03), Los Angels, CA, USA, pp. 40-50, 2003.

[9] L. Lazos, R. Poovendran, and J. A. Ritcey, "Probabilistic detection of mobile targets in heterogeneous sensor networks,” in Proceedings of IPSN’07, pp. 519-528, 2007.

[10] W. Z. Zhang, M. L. Li, and M. Y. Wu, “An algorithm for target traversing based on local Voronoi diagram,” Journal of Software, Vol. 18, No, 5, pp. 1246-1253, 2007.

[11] C. D. Jiang and G. L. Chen, "Double barrier coverage in dense sensor networks,” Journal of Computer Science and Technology, Vol. 23, No. 1, pp. 154-165, 2008.

[12] M. Cardei, M. T. Thai, Y. Li, and W. Wu, "Energy-efficient target coverage in wireless sensor networks,” Proceedings of IEEE INFOCOM'05, Miami, FL, pp. 1976-1983, 2005.

[13] M. Cardei, J. Wu, M. Lu, and M. O. Pervaiz, "Maximum network lifetime in wireless sensor networks with adjustable sensing ranges," Proceedings of IEEE International Conference on Wireless and Mobile Computing, Networking and Communications (WiMob’05), Montreal, Canada, pp. 1-8, 2005.

[14] S. Meguerdichian, F. Koushanfar, M. Potkonjak, and M. B. Srivastava, "Coverage problems in wireless ad-hoc sensor networks," in IEEE INFOCOM, Vol. 3, pp. 1380-1387, 2001. 
[15] S. Meguedrichian, S. Slijepcevic, V. Karayan, and M. Potkonjak, "Localized algorithms in wireless ad-hoc networks: Location discovery and sensor exposure," Proceedings of ACM MobiHoc'01, Long Beach, CA, USA, pp. 106-116, 2001.

[16] M. Lu, J. Wu, M. Cardei, and M. Li, "Energy-efficient connected coverage of discrete targets in wireless sensor networks," Proceedings of 2005 International Conference on Computer Networks and Mobile Computing (ICCNMC’05), Zhangjiajie, China, pp. 1-10, 2005.

[17] X. Li, P. Wan, and O. Frieder, "Coverage in wireless ad-hoc sensor networks,” Proceedings of IEEE International Conference on Communications (ICC'02), New York, NY, USA, pp. 1-5, 2002.

[18] S. Meguerdichian, F. Koushanfar, G. Qu, and M. Potkonjak, "Exposure in wireless ad hoc sensor networks," Proceedings of ACM MobiCom’01, pp. 139-150, 2001.

[19] S. Adlakha and M. Srivastava, "Critical density thresholds for coverage in wireless sensor networks,” Proceedings of IEEE Wireless Communications and Networking Conference (WCNC’03), NewOrleans, Louisiana, USA, pp. 16-20, 2003.

[20] B. Wang, et al., "Worst and best information exposure paths in wireless sensor networks," Proceedings of MSN 2005, pp. 52-62, December 2005.
[21] B. Liu and D. Towsley, "On the coverage and detectability of large-scale wireless sensor networks,” Proceedings of WiOpt'03: Modeling and Optimization in Mobile, Ad Hoc and Wireless Networks, INRIA Sophia-Antipolis, France, pp. 1-3, 2003.

[22] R. Meester and R. Roy, “Continuum percolation,” Balletin of the American Mathematical Society, Vol. 34, No. 4, pp. 447-448, 1997.

[23] B. Liu and D. Towsley, "A study of the coverage of large-scale sensor networks," Proceedings of the 1st IEEE International Conference on Mobile Ad-hoc and Sensor Systems (MASS’04), Florida, USA, pp. 475-481, 2004.

[24] S. Kumar, T. Lai, and A. Arora, "Barrier coverage with wireless sensors," Wireless Network, Vol. 13, pp. 817 834, 2007.

[25] S. Kloder and S. Hutchinson, "Barrier coverage for variable bounded-range line-of-sight guards," in Proceedings of IEEE International Conference on Robotics and Automation 2007, pp. 391-396, April 2007.

[26] J. O’Rourke, “Computational geometry: Column 15,” International Journal of Computational Geometry and Applications, Vol. 2, No. 2, pp. 215-217, 1992. 